\title{
SYNTHESIS AND REACTIONS OF SOME NEW MORPHOLINYLPYRROLYL TETRAHYDROTHIENO[2,3-c] ISOQUINOLINE
}

Remon M. Zaki\#, Adel M. Kamal El-Dean, Shaban M. Radwan

Chemistry department, Faculty of Science, Assiut University, Assiut 71516, Egypt

E-mail: remonch2003@yahoo.com

\section{ABSTRACT}

Hydrazinolysis of ethyl-5-morpholin-4-yl-1-(1H-pyrrol-1-yl)-6,7,8,9-tetrahydrothieno[2,3-c]isoquinoline-2-carboxylate afforded the corresponding carbohydrazide which upon condensation with aromatic aldehydes, acetyl acetone and/ or carbon disulfide gave $\mathrm{N}$ - arylidinecarbohydrazide, dimethylpyrazolyl methanone, [1,3,4]oxadiazole-2-thiol and its ethyl ester derivatives respectively. Diazotization of the carbohydrazide with nitrous acid afforded the corresponding carboazide which was used for synthesis of carbamates and substituted carboxamides. Boiling of the carboazide in dry xylene afforded the pyrazinone compound which was used for synthesis of other heterocycles containing pyrrolopyrazinothinoisoquinoline moeity.

KEYWORDS: Tetrahydrothienoisoquinoline, Pyrrole, Pyrazole, Triazole, Synthesis.

\section{Council for Innovative Research}

Peer Review Research Publishing System

Journal: Journal of Advances in Chemistry

Vol. 10, No. 3

editorjaconline@gmail.com

www.cirjac.com 


\section{INTRODUCTION}

Isoquinoline alkaloids are a large family of natural products and display a broad variety of biological activities. ${ }^{1}$ among the members of this class of compounds, tetrahydroisoquinoline derivatives constitute a major group. Many of them exhibit important biological activities, for example, anti-inflammatory, anti-microbial, anti-leukemic, and anti-tumor properties. ${ }^{2,3}$, anti-HIV, and other biological activities ${ }^{4-7}$. Substituted tetrahydroquinolines are the core structures in many important pharmacological agents and drug molecules such as anti-arrhythmic and cardiovascular agents, anticancer drugs, immunosuppressants and as ligands for $5-\mathrm{HTIA}$ and NMDA receptors ${ }^{8-11}$. Thienoquinolines are reported to exhibit a broad spectrum of biological effects. Some of them are useful as memory enhancers, ${ }^{12}$ antiallergics, ${ }^{13}$ anti-inflammatories, immunoregulators, analgesics and antipyritics. ${ }^{14}$ Others are known to possess a good antibacterial ${ }^{15}$ and antianaphylactic ${ }^{16}$ activities.

\section{RESULTS AND DISCUSSION}

In continuation of our work about synthesis of heterocyclic compounds containing morpholinyl- tetrahydrothieno[2,3c]isoquinoline moiety as described in references ${ }^{17-21}$ hoping these new compounds show biological activity. The authors incorporated pyrrolyl ring to the thienotetrahydroisoquinoline system through the reaction of ethyl-1-amino-5-morpholin-4yl-6,7,8,9-tetrahydrothino[2,3-c]isoquinole-2-carboxylate 1 with 2,5-dimethoxytetrahydrofuran in glacial acetic acid to afford the corresponding pyrrolyl ester 2 . The structure of compound 2 was established by IR, ${ }^{1} \mathrm{H}-\mathrm{NMR}$ and mass spectra. IR spectrum showed disappearance of absorption bands characteristic for $\mathrm{NH}_{2}$ group and absorption band at $1720 \mathrm{~cm}^{-1}$ for ester group is still remaining. ${ }^{1} \mathrm{H}-\mathrm{NMR}$ in $\mathrm{CDCl}_{3}$ of compound 2 showed singlet signal characteristic for pyrrolyl group at 6.30 and $6.70 \mathrm{ppm}$. Mass spectrum showed a peak at 411 as molecular ion peak and a base peak. Reaction of pyrrolyl ester $\mathbf{2}$ with hydrazine hydrate afforded the pyrrolyl carbohydrazide $\mathbf{3}$. The structure of $\mathbf{3}$ was elucidated by elemental and spectral analysis. IR spectrum showed absorption bands at 3400, 3500 and $3100 \mathrm{~cm}^{-1}$ characteristic for $\mathrm{NH}, \mathrm{NH}_{2}$ groups and lowering the wave number of $\mathrm{CO}$ group in hydrazide from $1720 \mathrm{~cm}^{-1}$ in ester compound 2 to $1645 \mathrm{~cm}^{-1}$ in carbohydrazide 3. ${ }^{1} \mathrm{H}$-NMR spectrum in DMSO- $\mathrm{d}_{6}$ showed signals at, 5.80 and 4.70 for $\mathrm{NH}_{2}, \mathrm{NH}$ groups respectively. Mass spectrum showed at peak at 397 as molecular ion peak.

The pyrrolyl carbohydrazide $\mathbf{3}$ was used as versatile precursor for synthesis of other hetero cyclic system. Thus, condensation of the carbohydrazide $\mathbf{3}$ with aromatic aldehydes namely benzaldhyde, $p$-anisaldehyde and/ or cinnamaldehyde afforded the corresponding Schiff's bases (imines) (4a-c). IR spectrum of 4a revealed disappearance of absorption bands characteristic for $\mathrm{NH}_{2}$ group in hydrazide 3. ${ }^{1} \mathrm{H}-\mathrm{NMR}$ spectrum in DMSO- $\mathrm{d}_{6}$ showed multiplet signals at $\delta$ 7.30-7.70 characteristic for aromatic protons and at 7.85 for $\mathrm{CH}$ benzylidene (scheme 1 ).

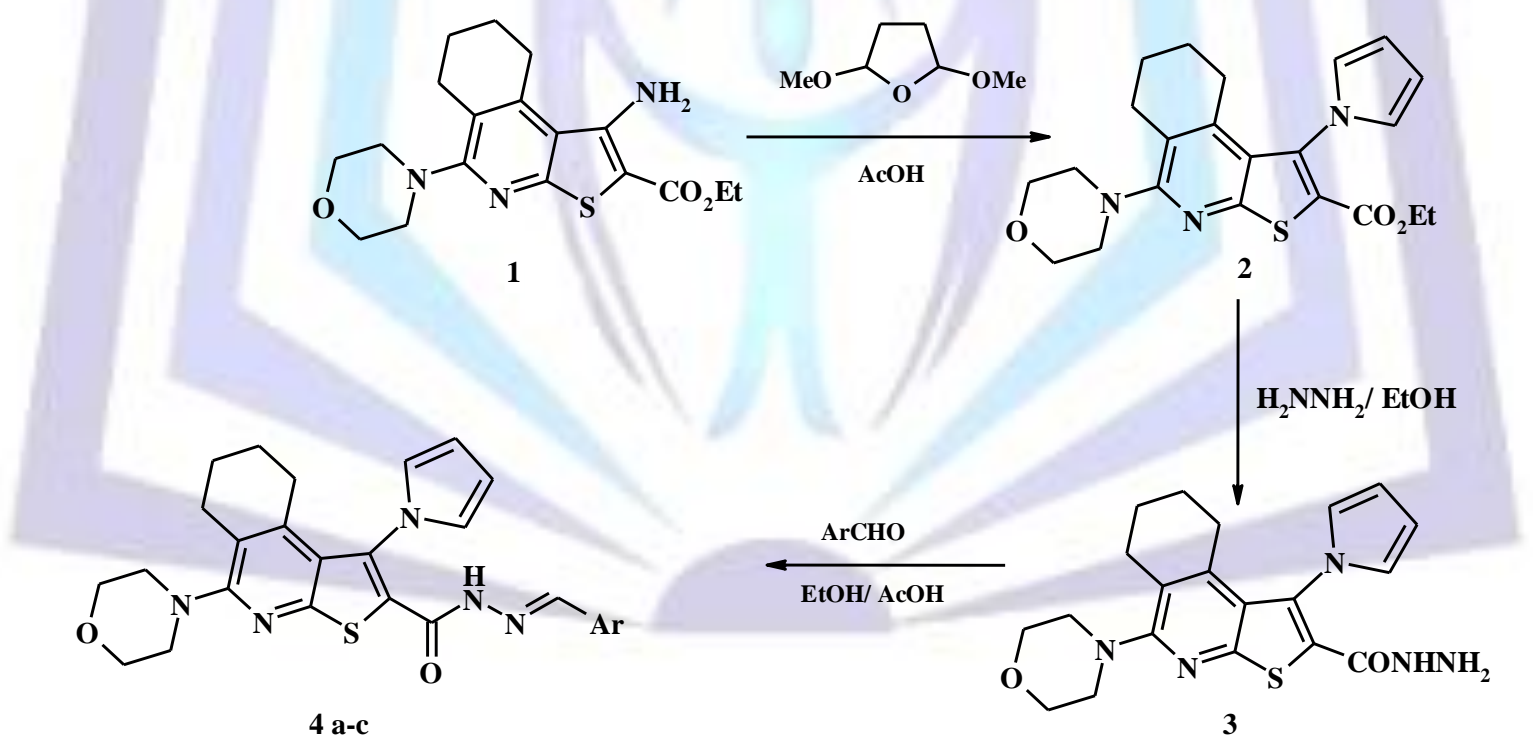

4a Ar: ph

Scheme 1

4b $\mathrm{Ar}: \operatorname{ph}\left(\mathrm{OCH}_{3}\right)-p$

4c Ar: $-\mathrm{CH}=\mathrm{CHph}$

Condensation of carbohydrazide $\mathbf{3}$ with acetyl acetone afforded the dimethylpyrazolyl derivative $\mathbf{5}$. The structure of the latter compound was established by elemental and spectral analysis. IR spectrum showed disappearance of absorption bands characteristic for $\mathrm{NH}, \mathrm{NH}_{2}$ group of carbohydrazide $3 .{ }^{1} \mathrm{H}-\mathrm{NMR}$ in $\mathrm{CDCl}_{3}$ showed two singlet signals at $\delta 2.30$ and 2.50 for two methyl groups of pyrazole and singlet signals at $5.95 \mathrm{ppm}$ for $\mathrm{CH}$ pyrazole. Also reaction of compound $\mathbf{3}$ with carbon disulfide in dry pyridine gave the oxadiazole thione 6 which was alkylated using ethyl chloroacetate in presence of ethanol and fused sodium acetate to afford the ethyl sulfanyl acetate 7 (scheme 2). 
<smiles>CCOC(=O)CSc1nnc(-c2sc3nc(N4CCOCC4)c4c(c3c2-n2cccc2)CCCC4)o1</smiles><smiles>NNC(=O)c1sc2nc(N3CCOCC3)c3c(c2c1-n1cccc1)CCCC3</smiles>

(1)

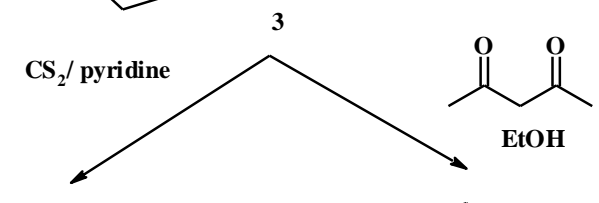<smiles>Sc1nnc(-c2sc3nc(N4CCOCC4)c4c(c3c2-n2cccc2)CCCC4)o1</smiles><smiles>Cc1cc(C)n(C(=O)c2sc3nc(N4CCOCC4)c4c(c3c2-n2cccc2)CCCC4)n1</smiles>

\section{Scheme 2}

Diazotization of carbohydrazide $\mathbf{3}$ with sodium nitrite in acetic acid at room temperature afforded the corresponding carboazide 8. The structure of carboazide 8 was proved by m.p., TLC and IR spectra. IR spectrum revealed disappearance of absorption bands for $\mathrm{NH}, \mathrm{NH}_{2}$ and appearance of absorption band at $2150 \mathrm{~cm}^{-1}$ characteristic for azido group. The carboazide compound $\mathbf{8}$ reacted with various primary, secondary and tertiary alcohols namely: ethanol, methanol, isopropanol and tert-butanol to give the corresponding carbamates 9a-d. The structure of ethyl carbamate $9 a$ showed absorption bands at 3230 and $1715 \mathrm{~cm}^{-1}$ characteristic for $\mathrm{NH}$ and $\mathrm{CO}$ carbamate respectively. ${ }^{1} \mathrm{H}-\mathrm{NMR}$ of ethyl carbamate 9 in $\mathrm{CDCl}_{3}$ showed triplet and quartet signals at $\delta 1.30$ and 4.20 for ethyl ester group and singlet signal at $\delta$ 9.30 for $\mathrm{NH}$ group. On the other hand, the carboazide 8 reacts with various cyclic secondary amines and/ or aromatic amines (primary and secondary) to afford the corresponding carboxamide derivatives 10a-f. The structure of phenyl urea derivative 10c was elucidated by elemental and spectral data. IR spectrum showed absorption bands at 3350,3280 for $2 \mathrm{NH}$ groups. ${ }^{1} \mathrm{H}-\mathrm{NMR}$ spectrum in DMSO- $\mathrm{d}_{6}$ showed multiplet signals at $\delta 8.70,9.80 \mathrm{ppm}$ characteristic for $\mathrm{NHph}$ and NHCO respectively (scheme 3$)$. 


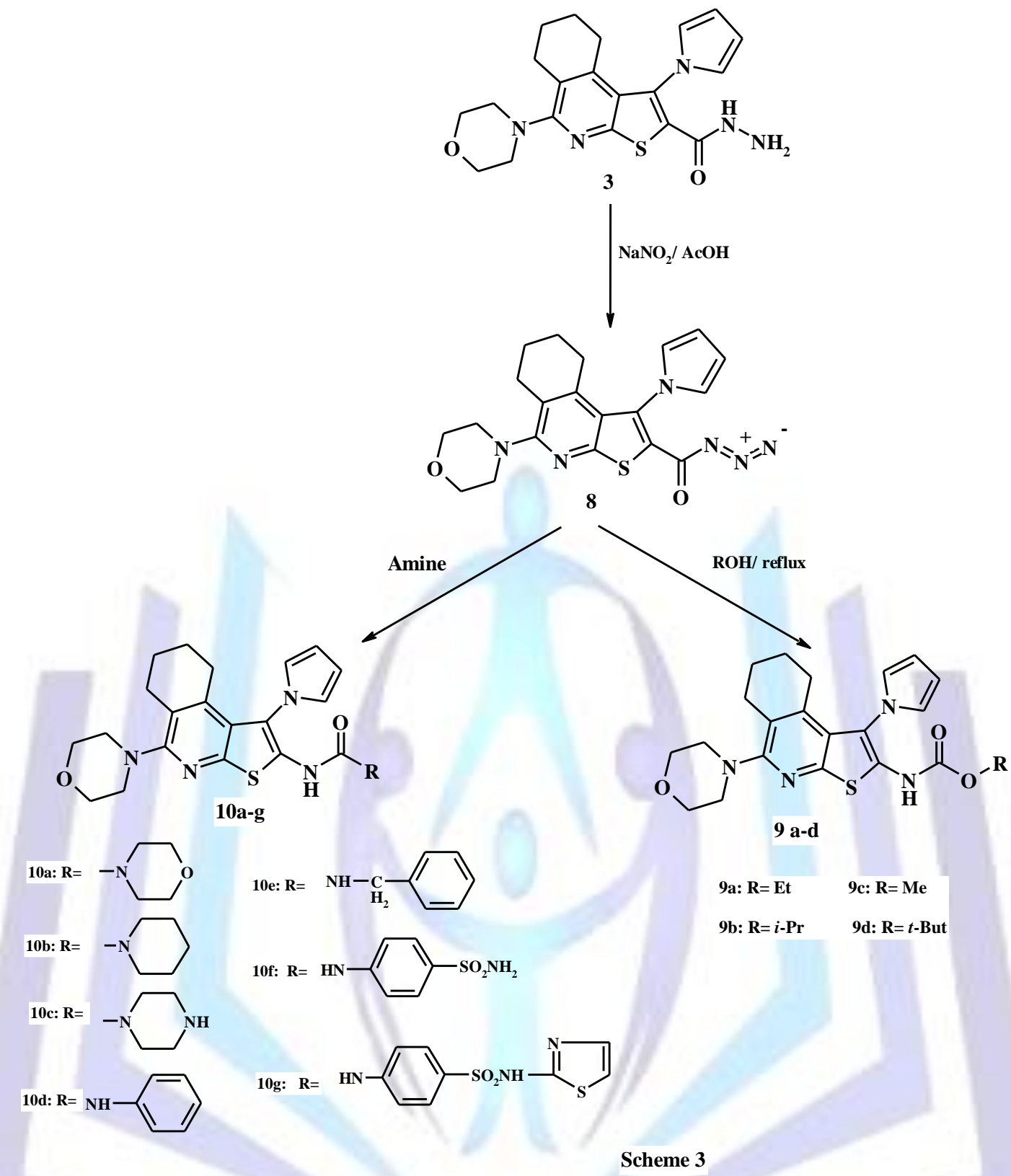

The carboazide 8 undewent Curtius rearrangement upon boiling dry xylene to afford the corresponding pyrrolopyrazinothienoisoquinoline 11. The structure of the latter compound $\mathbf{1 1}$ was established by elemental and spectral analysis. IR spectrum revealed the disappearance of absorption band at $2150 \mathrm{~cm}^{-1}$ characteristic for azido group and appearance of absorption band at $3280 \mathrm{~cm}^{-1}$ for $\mathrm{NH}$ group. ${ }^{1} \mathrm{H}-\mathrm{NMR}$ in $\mathrm{CF}_{3} \mathrm{CO}_{2} \mathrm{D}$ showed three singlet signals at $\delta 6.20$, 6.50 and $7.00 \mathrm{ppm}$ characteristic for the three $\mathrm{CH}$ pyrrolo groups.

Chlorination of the pyrazino compound $\mathbf{1 1}$ with phosphorus oxychloride under reflux gave the corresponding chloro derivative 12, which underwent nucleophilic substitution reactions with primary amines such as aniline and/or hydrazine hydrate to afford the corresponding phenyl amino 13 and hydrazino 14 respectively. The structure of compounds 13,14 was proved by IR, ${ }^{1} \mathrm{H}$-NMR spectra. IR spectrum of compound 13 showed absorption band at $3400 \mathrm{~cm}^{-1}$ for $\mathrm{NH}$ group. $1 \mathrm{H}$ NMR spectrum in DMSO- $\mathrm{d}_{6}$ showed multiplet signals at $\delta 7.20-7.80 \mathrm{ppm}$ characteristic for aromatic protons. While IR spectrum of hydrazine 14 showed absorption band at 3350,3300 and 3250 for $\mathrm{NH}, \mathrm{NH}_{2}$ groups. ${ }^{1} \mathrm{H}-\mathrm{NMR}$ of compound 14 in $\mathrm{CDCl}_{3}$ showed singlet signals at $\delta 6.50$ and $\delta 7.90$ for $\mathrm{NH}_{2}$ and $\mathrm{NH}$ groups respectively.

Reaction of hydrazino compound $\mathbf{1 4}$ with benzaldehyde afforded the corresponding benzylidene-8-morpholin-4-yl9,10,11,12-tetrahydropyrrolo[1",2":4',5']pyrazino[2',3': 5,4]thieno[2,3-c]isoquinoline-4-ylhydrazide (15), whilst condensation of hydrazino compound $\mathbf{1 4}$ with triethyl orthoformate in refluxing acetic acid accompanied by loss of ethanol molecule afforded the corresponding triazolo derivative 16. The structure of compounds 15, 16 was established by IR, ${ }^{1} \mathrm{H}-\mathrm{NMR}$ spectra. IR spectrum of compound 15 revealed disappearance of absorption bands characteristic for $\mathrm{NH}, \mathrm{NH} 2 \mathrm{groups}$. $1 \mathrm{H}$ NMR in $\mathrm{CF}_{3} \mathrm{CO}_{2} \mathrm{D}$ showed multiplet signals at $\delta 7.20-7.85 \mathrm{ppm}$ for aromatic protons, while IR spectrum of compound 16 showed absorption band at $1640 \mathrm{~cm}^{-1}$ for $\mathrm{C}=\mathrm{N}$. ${ }^{1} \mathrm{H}-\mathrm{NMR}$ spectrum of compound 16 showed singlet signal at $8.30 \mathrm{for} \mathrm{CH}$ triazole (scheme 4). 


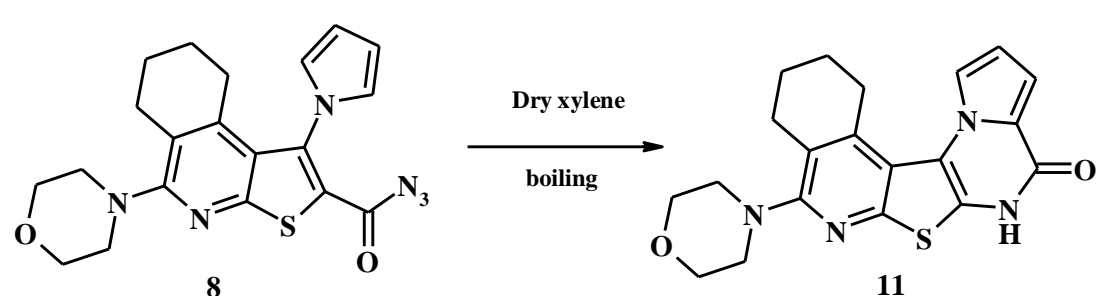

8

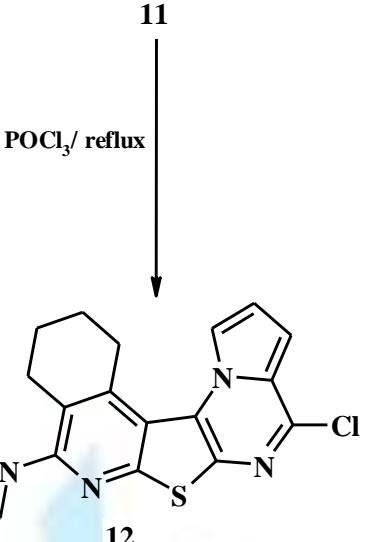

13
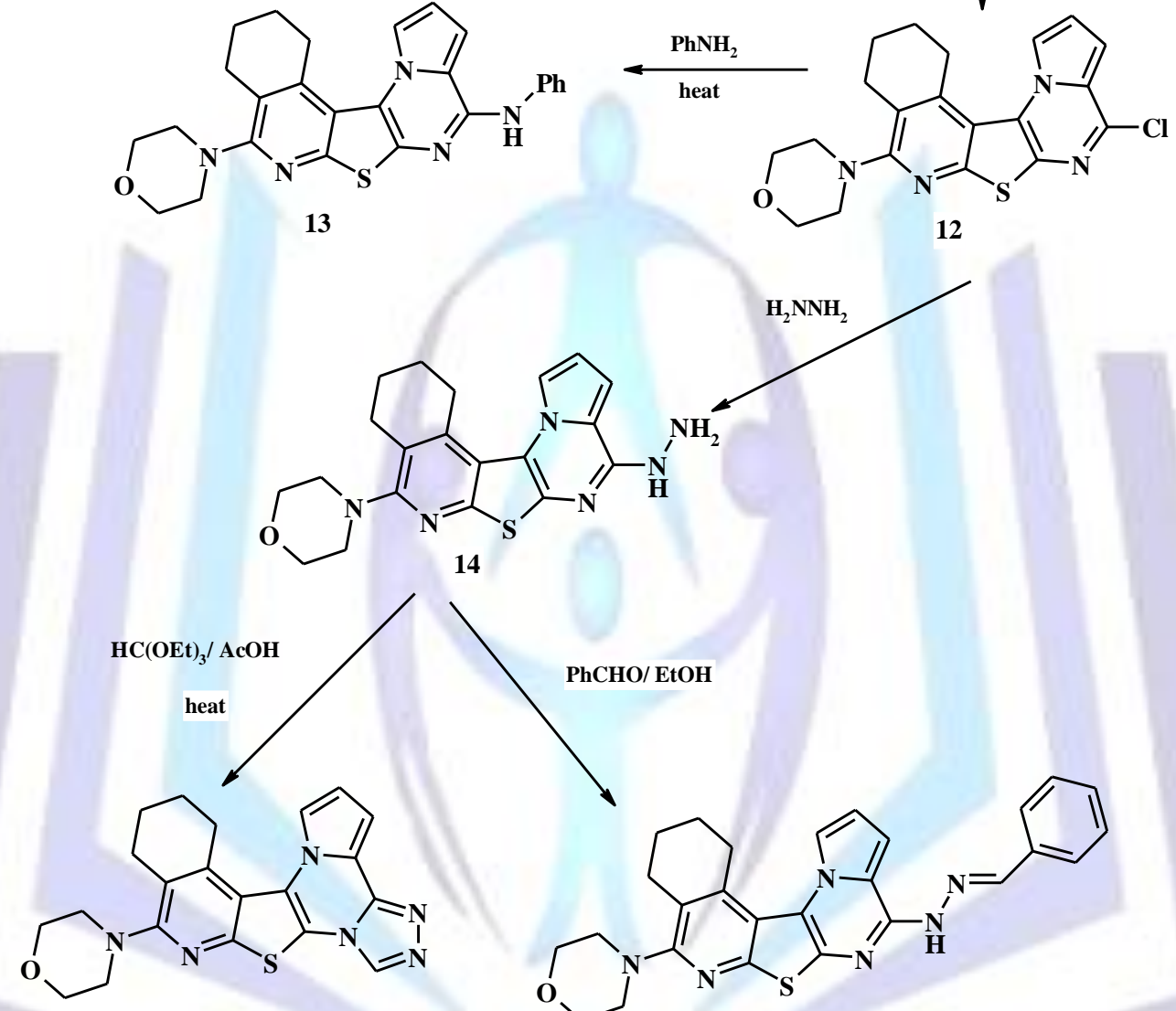

16

14
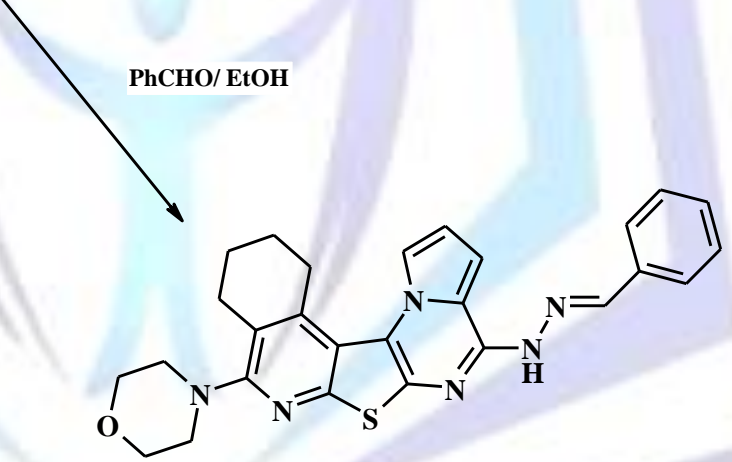

15

Scheme 4

Numbering of carbon atoms for compounds 2, 10a needed for ${ }^{13} \mathrm{C}-\mathrm{NMR}$ analysis are described in the following figure:

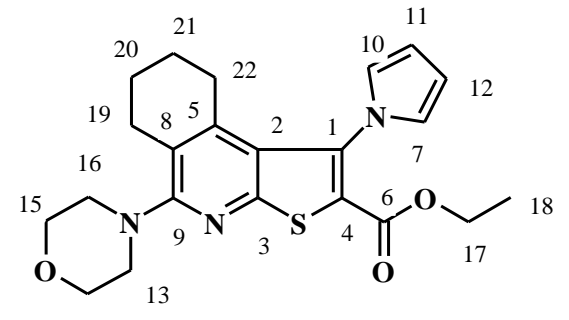

142

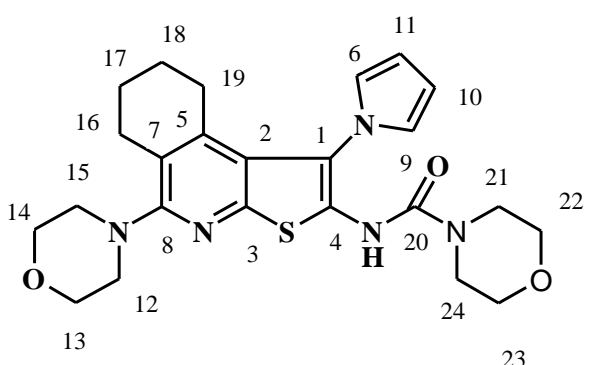

10a

Figure 1 


\section{EXPERIMENTAL}

All melting points are uncorrected and measured on a Fisher-John apparatus. Elemental analyses were determined on an Elementar Analysensystem GmbH-VarioEL V.3 microanalyzer in the central lab of Assiut University. Their results were found to be in good agreement $( \pm 0.2 \%)$ with the calculated values. IR spectra were recorded on a Pye-Unicam Sp-100 spectrophotometer using $\mathrm{KBr}$ wafer technique. NMR spectra were recorded on a varian EM-390 $90 \mathrm{MHz}$ and Joel 400 $\mathrm{MHz}$ spectrometers in a suitable deutrated solvent using TMS as internal standard (chemical shifts in ppm). MS spectra were recorded on Jeol JMS-600 apparatus. The amino ester compound $\mathbf{1}$ was prepared according to literature procedure ${ }^{17}$ with melting point $202-204^{\circ} \mathrm{C}$.

\section{Ethyl-5-morpholin-4-yl-1-(1H-pyrrol-1-yl)-6,7,8,9-tetrahydrothieno[2,3-c] isoquinoline-2- carboxylate (2)}

A mixture of ethyl-1-amino-5-morpholin-4-yl-6,7,8,9-tetrahydrothieno[2,3-c]iso quinoline-2-carboxylate (1) (3.6 g, $0.01 \mathrm{~mol}$ ) and 2,5-dimethoxytetrahydrofuran $(1.50 \mathrm{ml}, 0.011 \mathrm{~mol})$ in acetic acid $(15 \mathrm{ml})$ was refluxed for $1 \mathrm{hr}$. The solid product which formed on cold was filtered off, dried and recrystallized from ethanol to give white needles in $83 \%$ yield, m.p. $186-188^{\circ} \mathrm{C}$. Anal. Calcd. For: $\mathrm{C}_{22} \mathrm{H}_{25} \mathrm{~N}_{3} \mathrm{O}_{3} \mathrm{~S}$ (411.53): C, 64.21; H, 6.12; N, 10.21; S, 7.79\%. Found: C, 64.25; H, 6.10; N, 10.16; S, 7.83\%. IR v (cm ${ }^{-1}$ ): 2950, 2850 (CH aliphatic), 1720 (CO ester), $1620(\mathrm{C}=\mathrm{N}) .{ }^{1} \mathrm{H}-\mathrm{NMR}\left(\mathrm{CDCl}_{3}\right): 1.10-1.30$ (t, J= 9.00 Hz, 3H, $\left.\mathrm{CH}_{3} \mathrm{ester}\right), 1.70$ (m, $4 \mathrm{H}, 2 \mathrm{CH}_{2}$ cyclohexeno), $2.65\left(\mathrm{~m}, 4 \mathrm{H}, 2 \mathrm{CH}_{2}\right.$ cyclohexeno), $3.35\left(\mathrm{~m}, 4 \mathrm{H}, 2 \times \mathrm{CH}_{2}-\mathrm{N}\right.$ morpholine), $3.80\left(\mathrm{~m}, 4 \mathrm{H}, 2 \times \mathrm{CH}_{2}-\mathrm{O}\right.$ morpholine), $4.05-4.30$ (q, J=7.50 Hz, $2 \mathrm{H}, \mathrm{CH}_{2}$ ester), $6.30\left(\mathrm{~s}, 2 \mathrm{H}, 2 \mathrm{CH}=\mathrm{C}\right.$ pyrrolyl), $6.70\left(\mathrm{~s}, 2 \mathrm{H}, 2 \mathrm{CH}-\mathrm{N}\right.$ pyrrolyl). ${ }^{13} \mathrm{C}-\mathrm{NMR}$ $\left(\mathrm{CDCl}_{3}, 400 \mathrm{MHz}\right): 13.91$ (C18, CH3 ester), 22.03, 22.27, 22.32, 26.45 (C19-C22 cyclohexeno), $50.10\left(\mathrm{C} 13: \mathrm{C}^{2},\left(\mathrm{CH}_{2}\right)_{2}-\mathrm{N}\right.$ morpholino), 61.29 ( $\mathrm{C} 17, \mathrm{CH}_{2}$ ester), 66.96 (C14:C15, $\left(\mathrm{CH}_{2}\right)_{2}-\mathrm{O}$ morpholino), 109.15 (C11, C12 pyrrolyl), 122 (C7, C10 pyrrolyl), 124.90 (C2), 136.79 (C5), 144.96 (C1, C4), 156.03 (C3), 161 (C9), 161.64 (C6, CO ester). El-MS: $m / z(\%)=411$ (M+, 100), $381\left(\mathrm{M}^{+}\right.$-Et, 11), $366\left(\mathrm{M}^{+}-\mathrm{OEt}, 10\right), 354\left(\mathrm{M}^{+}\right.$-COEt, 22), $325\left(\mathrm{M}^{+}\right.$-morpholino, 4), 86 (morpholino, 3).

\section{5-Morpholin-4-yl-1-(1H-pyrrol-1-yl)-6,7,8,9-tetrahydrothieno[2,3-c]iso- quinoline-2-carbo hydrazide (3)}

A mixture of pyrrol-1-yl ester compound $2(1 \mathrm{~g}, 2.5 \mathrm{mmol})$ and hydrazine hydrate $(5 \mathrm{ml}, 0.1 \mathrm{~mol})$ was heated under neat conditions for $1 \mathrm{hr}$ then ethanol absolute $(10 \mathrm{ml})$ was added and reflux continued for additional $2 \mathrm{hrs}$. The solid product which formed on cold was filtered off, dried and recrystallized from ethanol to afford white crystals in $69 \%$ yield, m.p. $236-238^{\circ} \mathrm{C}$. Anal. Calcd. For: $\mathrm{C}_{20} \mathrm{H}_{23} \mathrm{~N}_{5} \mathrm{O}_{2} \mathrm{~S}(397.50) \mathrm{C}, 60.43 ; \mathrm{H}, 5.83 ; \mathrm{N}, 17.62 ; \mathrm{S}, 8.07 \%$. Found: C, 60.40; H, 5.79; N, 17.65; S, 8.00\%. IR $\vee\left(\mathrm{cm}^{-1}\right): 3400,3300,3100\left(\mathrm{NH}, \mathrm{NH}_{2}\right), 2950,2850\left(\mathrm{CH}\right.$ aliphatic), 1645 (CO hydrazide), $1620(\mathrm{C}=\mathrm{N}) .{ }^{1} \mathrm{H}-\mathrm{NMR}\left(\mathrm{DMSO}-\mathrm{d}_{6}\right)$ : 1.50 (m, 4H, $2 \times \mathrm{CH}_{2}$ cyclohexeno), 2.70 (m, 4H, $2 \times \mathrm{CH}_{2}$ cyclohexeno), 3.20 (m, $4 \mathrm{H}, 2 \times \mathrm{CH}_{2}-\mathrm{N}$ morpholine), 3.70 (m, $4 \mathrm{H}, 2 \times$ $\mathrm{CH}_{2}-\mathrm{O}$ morpholine), $5.80\left(\mathrm{~S}, 2 \mathrm{H}, \mathrm{NH}_{2}\right.$ ), 6.30 (s, 2H, 2CH=C pyrrrolyl), 6.95 (s, 2H, 2CH-N pyrrolyl), 7.40 (s, $\left.1 \mathrm{H}, \mathrm{NH}\right) . \mathrm{El}-\mathrm{MS}$ : $\mathrm{m} / \mathrm{z}(\%)=398\left(\mathrm{M}^{+}+1,20.1\right), 397\left(\mathrm{M}^{+}, 34\right), 396\left(\mathrm{M}^{+}-1,30\right), 368(22), 366\left(\mathrm{M}^{+}-\mathrm{N}_{2} \mathrm{H}_{3}, 100\right), 361(21), 353\left(\mathrm{M}^{+}-\mathrm{CONH}, 14\right), 297$ $\left(\mathrm{M}^{+}-\mathrm{C}_{4} \mathrm{H}_{8} \mathrm{~N}_{2} \mathrm{O}, 21\right), 281$ (17), 253 (23), 225 (16).

\section{N-Arylidene-1-(1H-pyrrol-1-yl)-5-morpholin-4-yl--6,7,8,9-tetrahydrothieno [2,3- c]isoquinoline-2-carbohydraide (4)}

\section{General procedure:}

Carbohydrazide compound $3(0.5 \mathrm{~g}, 2 \mathrm{mmol})$ and aromatic aldehyde $(2.5 \mathrm{mmol})$ were refluxed in ethanol $(20 \mathrm{ml})$ and acetic acid $(0.5 \mathrm{ml})$ for $2 \mathrm{hrs}$. The solid precipitate which is formed during reflux was filtered off, dried and recrystallized from the proper solvent.

\section{N-Benzylidene-1-(1H-pyrrol-1-yl)-5-morpholin-4-yl--6,7,8,9-tetrahydrothieno[2,3-c]isoquinoline-2- carbohydraide (4a)}

Obtained from carbohydrazide 3 and benzaldehyde. The solid precipitate which is formed during reflux was filtered off, dried and recrystallized from acetic acid as white crystals. IR v $\left(\mathrm{cm}^{-1}\right): 3300(\mathrm{NH}), 3050(\mathrm{CH}$ aromatic), $2950,2850(\mathrm{CH}$ aliphatic), 1650 (CO hydrazide), $1610(\mathrm{C}=\mathrm{N})$. ${ }^{1} \mathrm{H}-\mathrm{NMR}$ (DMSO- $\left.\mathrm{d}_{6}\right): 1.80\left(\mathrm{~m}, 4 \mathrm{H}, 2 \times \mathrm{CH}_{2}\right.$ cyclohexeno), $2.80(\mathrm{~m}, 4 \mathrm{H}, 2 \mathrm{x}$ $\mathrm{CH}_{2}$ cyclohexeno), 3.20 (m, $4 \mathrm{H}, 2 \times \mathrm{CH} 2-\mathrm{N}$ morpholine), 3.80 (m, $4 \mathrm{H}, 2 \times \mathrm{CH}_{2}-\mathrm{O}$ morphoine), 6.50 (s, $2 \mathrm{H}, 2 \mathrm{CH}=\mathrm{C}$ pyrrolyl), 6.95 (s, 2H, 2CH-N pyrrolyl), 7.30-7.70 (m, 5H, ArH), $7.85(\mathrm{~s}, 1 \mathrm{H}, \mathrm{N}=\underline{\mathrm{CH}} \mathrm{ph}), 8.00(\mathrm{~s}, 1 \mathrm{H}, \mathrm{NH})$. 
Table 1: Physical constants of compounds 4a-c, 9a-d, 10a-g

\begin{tabular}{|c|c|c|c|c|c|c|c|}
\hline \multirow[t]{2}{*}{ compound } & \multirow[t]{2}{*}{$\begin{array}{l}\text { Empirical } \\
\text { Formula }\end{array}$} & \multicolumn{4}{|c|}{$\frac{\text { Found, \% }}{\text { Calculated, \% }}$} & \multirow[t]{2}{*}{$\mathrm{Mp},{ }^{\circ} \mathrm{C}$} & \multirow[t]{2}{*}{ Yield } \\
\hline & & C & $\mathbf{H}$ & $\mathbf{N}$ & $\mathbf{S}$ & & \\
\hline $4 a$ & $\begin{array}{c}\mathrm{C}_{27} \mathrm{H}_{27} \mathrm{~N}_{5} \mathrm{O}_{2} \mathrm{~S} \\
(485.61)\end{array}$ & $\frac{66.72}{66.78}$ & $\frac{5.65}{5.60}$ & $\frac{14.38}{14.42}$ & $\frac{6.67}{6.60}$ & $260-262$ & $\begin{array}{l}0.44 \mathrm{~g} \\
(72 \%)\end{array}$ \\
\hline $4 b$ & $\begin{array}{c}\mathrm{C}_{28} \mathrm{H}_{29} \mathrm{~N}_{5} \mathrm{O}_{3} \mathrm{~S} \\
(515.64)\end{array}$ & $\frac{65.10}{65.22}$ & $\frac{5.71}{5.67}$ & $\frac{13.64}{13.58}$ & $\frac{6.28}{6.22}$ & $268-270$ & $\begin{array}{l}0.52 \mathrm{~g} \\
(80 \%)\end{array}$ \\
\hline 4c & $\begin{array}{c}\mathrm{C}_{29} \mathrm{H}_{29} \mathrm{~N}_{5} \mathrm{O}_{2} \mathrm{~S} \\
(511.62)\end{array}$ & $\frac{67.94}{68.08}$ & $\frac{5.80}{5.71}$ & $\frac{13.75}{13.69}$ & $\frac{6.33}{6.27}$ & $280-282$ & $\begin{array}{l}0.49 \mathrm{~g} \\
(76 \%)\end{array}$ \\
\hline $9 a$ & $\mathrm{C}_{22} \mathrm{H}_{26} \mathrm{~N}_{4} \mathrm{O}_{3} \mathrm{~S}$ & $\frac{62.00}{61.95}$ & $\frac{6.22}{6.14}$ & $\frac{13.24}{13.14}$ & $\frac{7.58}{7.52}$ & $232-234$ & $\begin{array}{l}0.43 \mathrm{~g} \\
(83 \%)\end{array}$ \\
\hline $9 b$ & $\mathrm{C}_{21} \mathrm{H}_{24} \mathrm{~N}_{4} \mathrm{O}_{3} \mathrm{~S}$ & $\frac{61.05}{61.15}$ & $\frac{5.90}{5.86}$ & $\frac{13.64}{13.58}$ & $\frac{7.85}{7.77}$ & $210-212$ & $0.4 \mathrm{~g}(80 \%)$ \\
\hline $9 c$ & $\mathrm{C}_{23} \mathrm{H}_{28} \mathrm{~N}_{4} \mathrm{O}_{3} \mathrm{~S}$ & $\frac{62.76}{62.70}$ & $\frac{6.50}{6.41}$ & $\frac{12.68}{12.72}$ & $\frac{7.40}{7.28}$ & $216-218$ & $\begin{array}{l}0.46 \mathrm{~g} \\
(86 \%)\end{array}$ \\
\hline $9 d$ & $\mathrm{C}_{24} \mathrm{H}_{30} \mathrm{~N}_{4} \mathrm{O}_{3} \mathrm{~S}$ & $\frac{63.48}{63.41}$ & $\frac{6.54}{6.65}$ & $\frac{12.25}{12.32}$ & $\frac{6.96}{7.05}$ & $226-228$ & $\begin{array}{l}0.42 \mathrm{~g} \\
(76 \%)\end{array}$ \\
\hline $10 a$ & $\mathrm{C}_{24} \mathrm{H}_{29} \mathrm{~N}_{5} \mathrm{O}_{3} \mathrm{~S}$ & $\frac{61.57}{61.65}$ & $\frac{6.00}{6.25}$ & $\frac{15.10}{14.98}$ & $\frac{6.92}{6.86}$ & $208-210$ & $\begin{array}{l}0.39 \mathrm{~g} \\
(68 \%)\end{array}$ \\
\hline $10 b$ & $\mathrm{C}_{24} \mathrm{H}_{30} \mathrm{~N}_{6} \mathrm{O}_{2} \mathrm{~S}$ & $\frac{61.82}{61.78}$ & $\frac{6.54}{6.48}$ & $\frac{17.92}{18.01}$ & $\frac{7.00}{6.86}$ & $224-226$ & $\begin{array}{l}0.34 \mathrm{~g} \\
(60 \%)\end{array}$ \\
\hline $10 c$ & $\mathrm{C}_{25} \mathrm{H}_{31} \mathrm{~N}_{5} \mathrm{O}_{2} \mathrm{~S}$ & $\frac{64.56}{64.49}$ & $\frac{6.66}{6.71}$ & $\frac{14.97}{15.04}$ & $\frac{7.00}{6.89}$ & $220-222$ & $\begin{array}{l}0.37 \mathrm{~g} \\
(64 \%)\end{array}$ \\
\hline $10 d$ & $\mathrm{C}_{26} \mathrm{H}_{27} \mathrm{~N}_{5} \mathrm{O}_{2} \mathrm{~S}$ & $\frac{66.00}{65.94}$ & $\begin{array}{l}\underline{5.62} \\
5.75\end{array}$ & $\frac{14.85}{14.79}$ & $\frac{6.70}{6.77}$ & $218-220$ & $\begin{array}{l}0.42 \mathrm{~g} \\
(70 \%)\end{array}$ \\
\hline $10 e$ & $\mathrm{C}_{27} \mathrm{H}_{29} \mathrm{~N}_{5} \mathrm{O}_{2} \mathrm{~S}$ & $\frac{66.62}{66.51}$ & $\frac{6.08}{5.99}$ & $\frac{14.47}{14.36}$ & $\frac{6.48}{6.58}$ & $230-232$ & $\begin{array}{l}0.48 \mathrm{~g} \\
(80 \%)\end{array}$ \\
\hline $10 f$ & $\mathrm{C}_{26} \mathrm{H}_{28} \mathrm{~N}_{6} \mathrm{O}_{4} \mathrm{~S}_{2}$ & $\frac{56.61}{56.50}$ & $\frac{5.20}{5.11}$ & $\frac{15.15}{15.21}$ & $\frac{11.73}{11.60}$ & $234-236$ & $\begin{array}{l}0.44 \mathrm{~g} \\
(65 \%)\end{array}$ \\
\hline
\end{tabular}




\begin{tabular}{|l|l|l|l|l|l|l|l|}
\hline $10 \mathrm{~g}$ & $\mathrm{C}_{29} \mathrm{H}_{29} \mathrm{~N}_{7} \mathrm{O}_{4} \mathrm{~S}_{3}$ & $\frac{54.86}{54.79}$ & $\frac{4.51}{4.60}$ & $\frac{15.36}{15.42}$ & $\frac{15.00}{15.13}$ & $238-240$ & $\begin{array}{l}0.44 \mathrm{~g} \\
(56 \%)\end{array}$ \\
\hline
\end{tabular}

N-(4-Methoxybenzylidene-1-(1H-pyrrol-1-yl)-5-morpholin-4-yl--6,7,8,9-tetra hydrothieno[2,3c]isoquinoline-2-carbohydraide (4b)

Obtained from carbohydrazide 3 and $p$-.anisaldehyde. The solid precipitate which is formed during reflux was filtered off, dried and recrystallized from acetic acid as white crystals. IR v $\left(\mathrm{cm}^{-1}\right): 3280(\mathrm{NH}), 3030(\mathrm{CH}$ aromatic), $2950,2850(\mathrm{CH}$ aliphatic), 1645 (CO hydrazide), $1600(\mathrm{C}=\mathrm{N}) .{ }^{1} \mathrm{H}-\mathrm{NMR}\left(\mathrm{CF}_{3} \mathrm{CO}_{2} \mathrm{D}\right): 1.95\left(\mathrm{~m}, 4 \mathrm{H}, 2 \times \mathrm{CH}_{2}\right.$ cyclohexeno), $2.90(\mathrm{~m}, 4 \mathrm{H}, 2 \mathrm{x}$ $\mathrm{CH}_{2}$ cyclohexeno), 3.90 (m, 4H, $2 \times \mathrm{CH}_{2}-\mathrm{N}$ morpholine), $4.25\left(\mathrm{~s}, 3 \mathrm{H}, \mathrm{OCH}_{3}\right), 4.50\left(\mathrm{~m}, 4 \mathrm{H}, 2 \times \mathrm{CH}_{2}-\mathrm{O}\right.$ morpholine), 6.95 (s, $2 \mathrm{H}, 2 \mathrm{CH}=\mathrm{C}$ pyrrolyl), 7.30 (s, 2H, 2CH-N pyrrolyl), 7.70-8.00 (m. $5 \mathrm{H}, \mathrm{ArH}), 8.25(\mathrm{~s}, 1 \mathrm{H}, \mathrm{N}=\mathrm{CH})$.

\section{2-Morholin-4-yl-N-(3-phenylallylidene)-1-(1H-pyrrol-1-yl)-6, 7,8,9-tetrahydro thieno[2,3-c] isoquinoline-2-carbohydrazide (4c)}

Obtained from carbohydrazide 3 and cinnamaldehyde. The solid precipitate which is formed during reflux was filtered off, dried and recrystallized from dioxane as yellow crystals. IR $\vee(\mathrm{cm}-1)$ : $3300(\mathrm{NH}), 3050$ (CH aromatic), 1655 (CO), 1600 $(\mathrm{C}=\mathrm{N}) .{ }^{1} \mathrm{H}-\mathrm{NMR}\left(\mathrm{CF}_{3} \mathrm{CO}_{2} \mathrm{D}\right): 1.80\left(\mathrm{~m}, 4 \mathrm{H}, 2 \times \mathrm{CH}_{2}\right.$ cyclohexeno), $2.80\left(\mathrm{~m}, 4 \mathrm{H}, 2 \times \mathrm{CH}_{2}\right.$ cyclohexeno), $3.30\left(\mathrm{~m}, 4 \mathrm{H}, 2 \times \mathrm{CH}_{2}-\right.$ $\mathrm{N}$ morpholine), 3.90 (m, $4 \mathrm{H}, 2 \times \mathrm{CH}_{2}-\mathrm{O}$ morpholine), 6.30 (s, $2 \mathrm{H}, 2 \times \mathrm{CH}=\mathrm{C}$ pyrrolyl), 6.90 (s, $2 \mathrm{H}, 2 \times \mathrm{CH}-\mathrm{N}$ pyrrolyl), $7.20-$ $7.80(\mathrm{~m}, 7 \mathrm{H}, \mathrm{ArH}+\mathrm{CH}=\mathrm{CH}), 8.30(\mathrm{~s}, 1 \mathrm{H}, \mathrm{N}=\mathrm{CH})$.

\section{(3,5-Dimethyl-1H-pyrazol-1-yl)(5-morpholino-1-(1H-pyrrol-1-yl)-6,7,8,9-tetrahydro thieno[2,3-c]isoquinolin-2-yl)methanone (5)}

A mixture of carbohydrazide compound $3(0.5 \mathrm{~g}, 1.2 \mathrm{mmol})$ and acetyl acetone $(0.2 \mathrm{ml}, 2 \mathrm{mmol})$ in ethanol $(20 \mathrm{ml})$ was refluxed for $3 \mathrm{hrs}$. The solid product which formed during reflux was filtered off, dried and recrystallized from ethanoldioxane mixture in $79 \%$ yield, m.p. $178-180^{\circ} \mathrm{C}$. Anal. Calcd. For: $\mathrm{C}_{25} \mathrm{H}_{27} \mathrm{~N}_{5} \mathrm{O}_{2} \mathrm{~S}$ (461.59) $\mathrm{C}, 65.05 ; \mathrm{H}, 5.90 ; \mathrm{N}, 15.17 ; \mathrm{S}$, 6.95\%. Found: C, 65.00; H, 6.00; N, 15.23; S, 7.00\%. IR v (cm $\left.{ }^{-1}\right): 3920,2820\left(\mathrm{CH}\right.$ aliphatic), $1695(\mathrm{CO}), 1620(\mathrm{C}=\mathrm{N}) .{ }^{1} \mathrm{H}-$ NMR $\left(\mathrm{CDCl}_{3}\right): 1.65\left(\mathrm{~m}, 4 \mathrm{H}, 2 \times \mathrm{CH}_{2}\right.$ cyclohexeno), 2.30, $2.50\left(2 \mathrm{~s}, 6 \mathrm{H}, 2 \times \mathrm{CH}_{3}\right.$ pyrazole), $2.70\left(\mathrm{~m}, 4 \mathrm{H}, 2 \times \mathrm{CH}_{2}\right.$ cyclohexeno), 3.25 (m, 4H,2 x CH $2-\mathrm{N}$ morpholine), 3.80 (m, 4H, $2 \times \mathrm{CH}_{2}-\mathrm{O}$ morpholine), 5.95 (s, $1 \mathrm{H}, \mathrm{CH}$ pyrazole), 6.35 (s, $2 \mathrm{H}, 2 \mathrm{CH}=\mathrm{C}$ pyrrolyl), 6.80 (s, 2H, CH-N pyrrolyl). El-MS: $m / z(\%)=462\left(\mathrm{M}^{+}+1,11\right), 461\left(\mathrm{M}^{+}, 100\right), 446\left(\mathrm{M}^{+}-\mathrm{CH}_{3}, 45\right), 431$ $\left(\mathrm{M}^{+}-2 \mathrm{CH}_{3}, 9.2\right), 397\left(\mathrm{M}^{+}-\mathrm{C}_{5} \mathrm{H}_{4}, 11\right), 375\left(\mathrm{M}^{+}\right.$-morpholino, 21.6), $366\left(\mathrm{M}_{+}-\mathrm{C}_{5} \mathrm{H}_{7} \mathrm{~N}_{2}, 4.6\right), 338\left(\mathrm{M}^{+}-\mathrm{C}_{6} \mathrm{H}_{7} \mathrm{~N}_{2} \mathrm{O}, 15\right), 252(\mathrm{M}+-$ $\left.\mathrm{C}_{10} \mathrm{H}_{15} \mathrm{~N}_{3} \mathrm{O}_{2}, 9\right)$.

\section{5-(5-Morpholin-4-yl-1-pyrrol-1-yl-6,7,8,9-tetrahydrothieno[2,3-c]iso quinolin-2-yl)- $[1,3,4]$ oxadiazole-2-thiol (6)}

A mixture of carbohydrazide compound $3(1.0 \mathrm{~g}, 1.20 \mathrm{mmol})$ and carbon disulfide $(2 \mathrm{ml})$ in dry pyridine $(4 \mathrm{ml})$ was heated on steam bath for $6 \mathrm{hrs}$. The solid product which formed during heating washed with ethanol, filtered off, dried and recrystallized from ethanol into pale yellow crystals in $81 \%$ yield, m.p. $312-314^{\circ} \mathrm{C}$. Anal. Calcd. For: $\mathrm{C}_{12} \mathrm{H}_{21} \mathrm{~N}_{5} \mathrm{O}_{2} \mathrm{~S}_{2}(439.56)$ C, 57.38; H, 4.82; N, 15.93; S, 14.59\%. Found: C, 57.30; H, 4.86; N, 16.00; S, 14.64\%. IR v $\left(\mathrm{cm}^{-1}\right): 2920,2850(\mathrm{CH}$ aliphatic), $1600(\mathrm{C}=\mathrm{N})$. ${ }^{1} \mathrm{H}-\mathrm{NMR}\left(\mathrm{CF}_{3} \mathrm{CO}_{2} \mathrm{D}\right): 2.00\left(\mathrm{~m}, 4 \mathrm{H}, 2 \times \mathrm{CH}_{2}\right.$ cyclohexeno), $2.80\left(\mathrm{~m}, 4 \mathrm{H}, 2 \times \mathrm{CH}_{2}\right.$ cyclohexeno), 3.65 (m, $4 \mathrm{H}, 2 \times \mathrm{CH}_{2}-\mathrm{N}$ morpholine), $4.20\left(\mathrm{~m}, 4 \mathrm{H}, 2 \times \mathrm{CH}_{2}-\mathrm{O}\right.$ morpholine), $6.70(\mathrm{~s}, 2 \mathrm{H}, 2 \mathrm{CH}=\mathrm{C}$ pyrrolyl), $7.05(\mathrm{~s}, 2 \mathrm{H}, 2 \mathrm{CH}-\mathrm{N}$ pyrrolyl).

\section{Ethyl[5-(5-Morpholin-4-yl-1-pyrrol-1-yl-6,7,8,9-tetrahydrothieno[2,3-c]isoquinolin-2-yl)- [1,3,4]oxadiazol-2-ylsulfanyl] acetate (7)}

A mixture of thiol compound $6(0.50 \mathrm{~g}, 1.13 \mathrm{mmol})$ and ethyl chloroacetate $(0.2 \mathrm{ml}, 1.6 \mathrm{mmol})$ in ethanol $(20 \mathrm{ml})$ in presence of fused sodium acetate $(0.3 \mathrm{~g}, 4 \mathrm{mmol})$ was refluxed for $2 \mathrm{hrs}$. The solid precipitate which formed on cooling and dilution with water was filtered off, dried and recrystallized from ethanol into white crystals in $76 \%$ yield, m.p. $224-$ $226^{\circ} \mathrm{C}$. Anal. Calcd. For: $\mathrm{C}_{25} \mathrm{H}_{27} \mathrm{~N}_{5} \mathrm{O}_{4} \mathrm{~S}_{2}(525.65) \mathrm{C}, 57.12 ; \mathrm{H}, 5.18 ; \mathrm{N}, 13.32 ; \mathrm{S}, 12.20 \%$. Found: C, 57.30; $\mathrm{H}, 5.38 ; \mathrm{N}$, 13.08; S, 12.23\%; IR v $\left(\mathrm{cm}^{-1}\right): 2950,2850$ (CH aliphatic), 1730 (CO ester), $1620(\mathrm{C}=\mathrm{N}) .{ }^{1} \mathrm{H}-\mathrm{NMR}\left(\mathrm{CDCl}_{3}\right): 1.30(\mathrm{t}, \mathrm{J}=7.50$ $\mathrm{Hz}, 3 \mathrm{H}, \mathrm{CH}_{3}$ ester), 1.70 (m, 4H, $2 \times \mathrm{CH}_{2}$ cyclohexeno), $2.70\left(\mathrm{~m}, 4 \mathrm{H}, 2 \times \mathrm{CH}_{2}\right.$ cyclohexeno), $3.20\left(\mathrm{~m}, 4 \mathrm{H}, 2 \times \mathrm{CH}_{2}-\mathrm{N}\right.$ morpholine), 3.80 (m, $4 \mathrm{H}, 2 \times \mathrm{CH}_{2}-\mathrm{O}$ morpholine), 4.10 (s, 2H, SCH ), 4.20 (q, J=6.00 Hz, 2H, $\mathrm{CH}_{2}$ ester), 6.30 (s, $2 \mathrm{H}$, $2 \mathrm{CH}=\mathrm{C}$ pyrrolyl), 6.90 (s, 2H, 2CH-N pyrrolyl).

\section{Morpholin-4-yl-1-(1H-pyrrol-1-yl)-6,7,8,9-tetrahydrothieno[2,3-c]iso quinoline-2- carboazide (8)}

Sodium nitrite solution $(0.30 \mathrm{~g}, 4.20 \mathrm{mmol}, 10 \%)$ was added dropwise with stirring to a solution of carbohydrazide compound $\mathbf{3}$ $(0.50 \mathrm{~g}, 1.20 \mathrm{mmol})$ in glacial acetic acid $(20 \mathrm{ml})$ at $0^{\circ} \mathrm{C}$ in an ice bath for 5 minutes. The solid product which formed during stirring was filtered off, dried and used without recrystallization in $56 \%$ yield, m.p. $140-142^{\circ} \mathrm{C}$. Anal. Calcd. $\mathrm{For}: \mathrm{C}_{20} \mathrm{H}_{20} \mathrm{~N}_{6} \mathrm{O}_{2} \mathrm{~S}$ (408.49) C, 58.81; H, 4.94; N, 20.57; S, 7.85\%. IR v $\left(\mathrm{cm}^{-1}\right): 2950,2850\left(\mathrm{CH}\right.$ aliphatic), $2150\left(\mathrm{~N}_{3}\right), 1665(\mathrm{CO}$ azide), 1580 $(\mathrm{C}=\mathrm{N})$. 


\section{Alkyl-5-morpholino-1-(1H-pyrrol-1-yl)-6,7,8,9-tetrahydrothieno[2,3-c]isoquinolin-2-yl carbamate (9 a-d)}

\section{General procedure:}

A solution of carboazide compound $8(0.5 \mathrm{~g}, 1.2 \mathrm{mmol})$ in an alcohol $(20 \mathrm{ml})$ was refluxed for $2 \mathrm{hrs}$. The solid product which formed during reflux was filtered off, dried and recrystallized from ethanol-dioxane 1:1.

\section{Ethyl-5-morpholino-1-(1H-pyrrol-1-yl)-6,7,8,9-tetrahydrothieno[2,3-c]iso quinolin-2-yl carbamate (9a)}

Obtained from carboazide 8 and ethanol. The solid product was recrystallized from ethanol-dioxane 1:1 mixture as white crystals. IR v $\left(\mathrm{cm}^{-1}\right): 3230(\mathrm{NH}), 2920,2820\left(\mathrm{CH}\right.$ aliphatic), 1715 (CO carbamate), $1590(\mathrm{C}=\mathrm{N}) .{ }^{1} \mathrm{H}-\mathrm{NMR}\left(\mathrm{CDCl}_{3}\right): 1.30(\mathrm{t}, \mathrm{J}=$

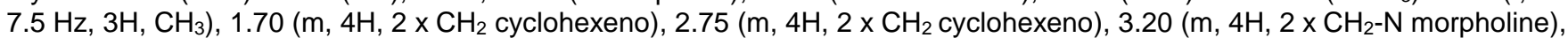
$3.80\left(\mathrm{~m}, 4 \mathrm{H}, 2 \times \mathrm{CH}_{2}-\mathrm{O}\right.$ morpholine), 4.20 (q, J=6.0 Hz, 2H, $\mathrm{CH}_{2}$ ester), 6.30 (s, 2H, 2CH=C pyrrolyl), 6.90 (s, $2 \mathrm{H}, 2 \mathrm{CH}-\mathrm{N}$ pyrrolyl), 9.30 (s, 1H, NH).

\section{Methyl-5-morpholino-1-(1H-pyrrol-1-yl)-6,7,8,9-tetrahydrothieno[2,3-c]isoquinolin-2-yl carbamate (9b)}

Obtained from carboazide 8 and methanol. The solid product was recrystallized from ethanol as pale brown crystals. IR $v(\mathrm{~cm}$ $\left.{ }^{1}\right): 3200(\mathrm{NH}), 2920,2850$ ( $\mathrm{CH}$ aliphatic), 1720 (CO ester), $1600(\mathrm{C}=\mathrm{N}) .{ }^{1} \mathrm{H}-\mathrm{NMR}\left(\mathrm{CDCl}_{3}\right): 1.50\left(\mathrm{~m}, 4 \mathrm{H}, 2 \times \mathrm{CH}_{2} \mathrm{cyclohexeno}\right.$ ), $2.60\left(\mathrm{~m}, 4 \mathrm{H}, 2 \times \mathrm{CH}_{2}\right.$ cyclohexeno), $3.00\left(\mathrm{~m}, 4 \mathrm{H}, 2 \times \mathrm{CH}_{2}-\mathrm{N}\right.$ morpholine), $3.70\left(\mathrm{~m}, 4 \mathrm{H}, 2 \times \mathrm{CH}_{2}-\mathrm{O}\right.$ morpholine), 6.25 (s, $2 \mathrm{H}$, $2 \mathrm{CH}=\mathrm{C}$ pyrrolyl), 6.70 (s, 2H, 2CH-N pyrrolyl), $9.60(\mathrm{~s}, 1 \mathrm{H}, \mathrm{NH})$.

Isopropyl-5-morpholino-1-(1H-pyrrol-1-yl)-6,7,8,9-tetrahydrothieno[2,3-c]isoquinolin-2-yl carbamate (9c)

Obtained from carboazide 8 and isopropanol. The solid product was recrystallized from ethanol-dioxane 1:1 mixture into pale red crystals. IR v $\left(\mathrm{cm}^{-1}\right): 3250(\mathrm{NH}), 2980,29220,2850$ ( $\mathrm{CH}$ aliphatic), 1715 (CO carbamate). 1H-NMR $(\mathrm{CDCl}): 1.30,1.35(\mathrm{~d}$, $6 \mathrm{H}, 2 \mathrm{CH}_{3}$ isopropyl), $1.70\left(\mathrm{~m}, 4 \mathrm{H}, 2 \times \mathrm{CH}_{2}\right.$ cyclohexeno), $2.70\left(\mathrm{~m}, 4 \mathrm{H}, 2 \times \mathrm{CH}_{2}\right.$ cyclohexeno), $3.15\left(\mathrm{~m}, 4 \mathrm{H}, 2 \times \mathrm{CH}_{2}-\mathrm{N}\right.$ morpholine), 3.80 (m, 4H, $2 \times \mathrm{CH}_{2}-\mathrm{O}$ morpholine), 5.00 (s, $1 \mathrm{H}, \mathrm{CH}$ isopropyl), 6.30 (s, 2H, 2CH=C pyrrolyl), $6.70(\mathrm{~s}, 2 \mathrm{H}, \mathrm{CH}-\mathrm{N}$ pyrrolyl), $9.55(\mathrm{~s}, 1 \mathrm{H}, \mathrm{NH})$. El-MS: $m / z(\%)=440(\mathrm{M}+, 100), 412(\mathrm{M}+-\mathrm{C} 2 \mathrm{H} 4,12), 397\left(\mathrm{M}^{+}-\mathrm{C}_{3} \mathrm{H}_{7}, 15\right), 381\left(\mathrm{M}^{+}-\mathrm{C}_{3} \mathrm{H}_{7} \mathrm{O}, 3\right), 354$ $\left(\mathrm{M}^{+}-\mathrm{C}_{4} \mathrm{H}_{6} \mathrm{O}_{2}, 21\right), 338\left(\mathrm{M}^{+}-\mathrm{C}_{4} \mathrm{H}_{8} \mathrm{NO}_{2}\right)$.

t-Butyl-(5-Morpholin-4-yl-1-pyrrol-1-yl-6,7,8,9-tetrahydrothieno[2,3-c]isoquinolin-2-yl) carbamate (9d)

Obtained by the reaction of carboazide 8 with tert-butanol. The solid product was recrystallized from ethanol-dioxane $1: 1$ mixture into white crystals. IR v $\left(\mathrm{cm}^{-1}\right): 3300(\mathrm{NH}), 2920,28510\left(\mathrm{CH}\right.$ aliphatic), 1710 (CO carbamate), $1590(\mathrm{C}=\mathrm{N}) .{ }^{1} \mathrm{H}-\mathrm{NMR}$ $\left(\mathrm{CDCl}_{3}\right): 1.50\left(\mathrm{~s}, 9 \mathrm{H}, 3 \mathrm{CH}_{3}\right), 1.70\left(\mathrm{~m}, 4 \mathrm{H}, 2 \times \mathrm{CH}_{2}\right.$ cyclohexeno), $2.70\left(\mathrm{~m}, 4 \mathrm{H}, 2 \times \mathrm{CH}_{2}\right.$ cyclohexeno), $3.10\left(\mathrm{~m}, 4 \mathrm{H}, 2 \times \mathrm{CH}_{2}-\mathrm{N}\right.$ morpholine), 3.85 (m, 4H, $2 \times \mathrm{CH}_{2}-\mathrm{O}$ morpholine), 6.30 (s, 2H, 2CH=C pyrroyl), 6.70 (s, 2H, 2CH-N pyrrolyl), 9.60 (s, $1 \mathrm{H}, \mathrm{NH}$ ).

\section{N-(5-Morpholino-1-(1H-pyrrol-1-yl)-6,7,8,9-tetrahydrothieno[2,3-c]iso- quinolin-2-yl) substituted-4-carboxamide (10a-g)}

\section{General procedure:}

A mixture of pyrrol-1-ylcarboazide $8(0.5 \mathrm{~g}, 1.2 \mathrm{mmol})$ and primary (secondry) amine $(1.25 \mathrm{mmol})$ was refluxed in dry toluene for $2 \mathrm{hrs}$. The solid product which formed on cooling was filtered off, dried and recrystallized from the proper solvent.

N-(5-Morpholino-1-(1H-pyrrol-1-yl)-6, 7,8,9-tetrahydrothieno[2,3-c]isoquinolin-2-yl) morpholine-4carboxamide (10a)

Obtained from carboazide 8 and morpholine. The solid product was recrystallized from ethanol as pale green crystals. IR v $\left(\mathrm{cm}^{-1}\right)$ : 3400-3300 (br NH), 2920, 2850 ( $\mathrm{CH}$ aliphatic), 1640 (CO amide). ${ }^{1} \mathrm{H}-\mathrm{NMR}\left(\mathrm{CDCl}_{3}\right): 1.80$ (m, 4H, $2 \times \mathrm{CH}_{2}$ cyclohexeno), $2.75\left(\mathrm{~m}, 4 \mathrm{H}, 2 \times \mathrm{CH}_{2}\right.$ cyclohexeno), $3.20\left(\mathrm{~m}, 8 \mathrm{H}, 4 \mathrm{CH}_{2}-\mathrm{N}\right.$ morpholine), $3.90\left(\mathrm{~m}, 8 \mathrm{H}, 4 \mathrm{CH}_{2}-\mathrm{O}\right.$ morpholine), $6.30(\mathrm{~s}, 2 \mathrm{H}, 2 \mathrm{CH}=\mathrm{C}$ pyrrolyl), 6.75 (s, 2H, 2CH-N pyrrolyl), $9.50(\mathrm{~s}, 1 \mathrm{H}, \mathrm{NH}) .{ }^{13} \mathrm{C}-\mathrm{NMR}\left(\mathrm{CDCl}_{3}\right)$ : 21.93, 22.27, 23.33, 26.45 (C16-C19 cyclohexeno), 50.31 (C12, C15, C21, C24 :2 x ( $\left.\mathrm{CH}_{2}\right)_{2}-\mathrm{N}$ morpholino), 66.52, 66.96 (C13, C14, C22, C23: $2 \times\left(\mathrm{CH}_{2}\right)_{2}-\mathrm{O}$ morpholino), 109.69 (C10, C11 pyrrolyl), 123 (C6, C9 pyrrolyl), 123.76 (C2), 129.87 (C5), 144.51 (C3, C4), 160.46 (C8), 161.30 (C20, CO). El-MS: $\mathrm{m} / \mathrm{z}(\%)=467.18(\mathrm{M}+, 10), 441(100)$.

\section{$\mathrm{N}$-(5-Morpholino-1-(1H-pyrrol-1-yl)-6, 7,8,9-tetrahydrothieno[2,3-c]isoquinolin-2-yl) piperazine-4- carboxamide (10b)}

Obtained from carboazide 8 and piperazine. The solid product was recrystallized from ethanol as white crystals. IR $v\left(\mathrm{~cm}^{-1}\right)$ : 3400, $3300(2 \mathrm{NH}), 2920,2850$ ( $\mathrm{CH}$ aliphatic), $1650(\mathrm{CONH}), 1580(\mathrm{C}=\mathrm{N}) .{ }^{1} \mathrm{H}-\mathrm{NMR}$ (DMSO-d 6 ): $1.90\left(\mathrm{~m}, 4 \mathrm{H}, 2 \mathrm{x} \mathrm{CH}_{2}\right.$ cyclohexeno), $2.60\left(\mathrm{~m}, 4 \mathrm{H}, 2 \times \mathrm{CH}_{2}\right.$ cyclohexeno), $2.90\left(\mathrm{~m}, 4 \mathrm{H}, 2 \mathrm{CH}_{2}-\mathrm{NH}\right.$ piperazine), $3.20\left(\mathrm{~m}, 8 \mathrm{H},\left(\mathrm{CH}_{2}\right)_{2}-\mathrm{N}\right.$ piperazine + $\left(\mathrm{CH}_{2}\right)_{2}-\mathrm{N}$ morpholine), 3.80 (m, 4H, $\left(\mathrm{CH}_{2}\right)_{2}-\mathrm{O}$ morpholine), 6.70 (s, 2H, 2CH=C pyrrolyl), 6.95 (s, 2H, 2CH-N pyrrolyl), 7.40 (s, $1 \mathrm{H}, \mathrm{NH}$ piperazine), 9.40 (s, $1 \mathrm{H}, \mathrm{NH}$ amide).

\section{N-(5-Morpholino-1-(1H-pyrrol-1-yl)-6,7,8,9-tetrahydrothieno[2,3-c]isoquinolin-2-yl) piperidine-4- carboxamide (10c)}

Obtained from carboazide 8 and piperidine. The solid product was recrystallized from ethanol as white $\mathrm{crystals} \mathrm{IR} \vee\left(\mathrm{cm}^{-1}\right)$ : $3400(\mathrm{NH}), 2950,2850$ ( $\mathrm{CH}$ aliphatic), 1630 (CO amide), $1560(\mathrm{C}=\mathrm{N}) .{ }^{1} \mathrm{H}-\mathrm{NMR}\left(\mathrm{CDCl}_{3}\right): 1.50\left(\mathrm{~m}, 4 \mathrm{H}, 2 \times \mathrm{CH}_{2} \mathrm{C} 2, \mathrm{C} 4\right.$ 
piperidine), 1.60 ( $\mathrm{m}, 2 \mathrm{H}, \mathrm{CH}_{2} \mathrm{C} 3$ piperidine), 1.75 ( $\mathrm{m}, 4 \mathrm{H}, 2 \times \mathrm{CH}_{2}$ cyclohexeno), 2.70 ( $\mathrm{m}, 4 \mathrm{H}, 2 \times \mathrm{CH}_{2}$ cyclohexeno), 3.20 ( $\mathrm{m}$, $4 \mathrm{H}, 2 \times \mathrm{CH}_{2}-\mathrm{N}$ morpholine), 3.40 (m, 4H, $2 \times \mathrm{CH}_{2} \mathrm{C}$, , C5 piperidine), 3.80 (m, $4 \mathrm{H}, 2 \times \mathrm{CH}_{2}-\mathrm{O}$ morpholine), 6.30 (s, $2 \mathrm{H}, 2 \mathrm{CH}=\mathrm{C}$ pyrrolyl), 6.90 (s, 2H, 2CH-N pyrrolyl), 9.60 (s, 1H, NH).

\section{1-(5-Morpholin-4-yl-1-(1H-pyrrol-1-yl)-6,7,8,9-tetrahydrothieno[2,3-c]isoquinolin-2-yl)-3-phenylurea (10d)}

Obtained from carboazide 8 and aniline. The solid product was recrystallized from dioxane as white needles. IR $\vee\left(\mathrm{cm}^{-1}\right): 3350$, $3280(2 \mathrm{NH}), 3030$ ( $\mathrm{CH}$ aromatic), 2920, 2850 (CH aliphatic), 1690 (CO), $1590(\mathrm{C}=\mathrm{N}) .{ }^{1} \mathrm{H}-\mathrm{NMR}\left(\mathrm{DMSO}-\mathrm{d}_{6}\right): 1.70(\mathrm{~m}, 4 \mathrm{H}, 2 \mathrm{x}$ $\mathrm{CH}_{2}$ cyclohexeno), 2.65 ( $\mathrm{m}, 4 \mathrm{H}, 2 \times \mathrm{CH}_{2}$ cyclohexeno), $3.25\left(\mathrm{~m}, 4 \mathrm{H}, 2 \times \mathrm{CH}_{2}-\mathrm{N}\right.$ morpholine), $3.70\left(\mathrm{~m}, 4 \mathrm{H}, 2 \times \mathrm{CH}_{2}-\mathrm{O}\right.$ morpholine), 6.30 (s, 2H, $2 \times \mathrm{CH}=\mathrm{C}$ pyrrolyl), 6.85 (s, 2H, $2 \times \mathrm{CH}-\mathrm{N}$ pyrrolyl), 7.30-7.70 (m, 5H, ArH), 8.70 (s, $1 \mathrm{H}, \mathrm{NHph}), 9.80$ (s, $1 \mathrm{H}, \mathrm{NHCO})$.

\section{1-Benzyl-3-(5-morpholin-4-yl-1-(1H-pyrrol-1-yl)-6,7,8,9-tetrahydrothieno[2,3-c]iso quinolin-2-yl)urea (10e)}

Obtained from carboazide 8 and benzyl amine. The solid product was recrystallized from ethanol-dioxane 1:1 mixture as yellow needles. IR v ( $\left.\mathrm{cm}^{-1}\right)$ : 3400, $3300(2 \mathrm{NH}), 3020$ ( $\mathrm{CH}$ aliphatic), 2920, 2850 ( $\mathrm{CH}$ aliphatic), $1630(\mathrm{CO}) .{ }^{1} \mathrm{H}_{-} \mathrm{NMR}(\mathrm{CDCl})$ ): $1.70\left(\mathrm{~m}, 4 \mathrm{H}, 2 \times \mathrm{CH}_{2}\right.$ cyclohexeno), 2.70 (m, $4 \mathrm{H}, 2 \times \mathrm{CH}_{2}$ cyclohexeno), $3.20\left(\mathrm{~m}, 4 \mathrm{H}, 2 \times \mathrm{CH}_{2}-\mathrm{N}\right.$ morpholine ), $3.90(\mathrm{~m}, 4 \mathrm{H}, 2 \times$ $\mathrm{CH}_{2}-\mathrm{O}$ morpholine), 4.30 (s, $2 \mathrm{H}, \mathrm{CH}_{2}$ ph), 6.30 (s, $2 \mathrm{H}, 2 \times \mathrm{CH}=\mathrm{C}$ pyrrolyl), 6.80 (s, $2 \mathrm{H}, 2 \times \mathrm{CH}-\mathrm{N}$ pyrrolyl), $7.10-7.40$ (m, $5 \mathrm{H}$, ArH), 8.00 (s, 1H, NH benzyl), 9.60 (s, 1H, NHCO).

\section{4-(3-(5-Morpholin-4-yl-1-(1H-pyrrol-1-yl)-6, 7,8,9-tetrahydrothieno[2,3-c]isoquinolin -2-yl) ureido)benzenesulfonamide (10f)}

Obtained from carboazide 8 and sulfanilamide. The solid product was recrystallized from dioxane as pale brown needles. IR $\mathrm{v}$ $\left(\mathrm{cm}^{-1}\right)$ : 3400, 3350, $3250\left(\mathrm{NH}, \mathrm{NH}_{2}\right), 3030$ ( $\mathrm{CH}$ aromatic), 2920, 2850 ( $\mathrm{CH}$ aliphatic), $1695(\mathrm{CO}) .{ }^{1} \mathrm{H}-\mathrm{NMR}$ (DMSO-d $)$ : $1.70(\mathrm{~m}$, $4 \mathrm{H}, 2 \times \mathrm{CH}_{2}$ cyclohexeno), $2.70\left(\mathrm{~m}, 4 \mathrm{H}, 2 \times \mathrm{CH}_{2}\right.$ cyclohexeno), $3.20\left(\mathrm{~m}, 4 \mathrm{H}, 2 \times \mathrm{CH}_{2}-\mathrm{N}\right.$ morpholine), $3.80\left(\mathrm{~m}, 4 \mathrm{H}, 2 \times \mathrm{CH}_{2}-\mathrm{O}\right.$ morpholine), 5.90 (s, 2H, $\mathrm{NH}_{2}$ ), 6.20 (s, 2H, $2 \times \mathrm{CH}=\mathrm{C}$ pyrrolyl), 6.80 (s, 2H, $2 \times \mathrm{CH}-\mathrm{N}$ pyrrolyl), 8.85 (s, $1 \mathrm{H}, \mathrm{NHph}$ ), 9.60 (s, $1 \mathrm{H}, \mathrm{CONH})$.

\section{4-(3-(5-Morpholin-4-yl)-1-(1H-pyrrol-1-yl)-6,7,8,9-tetragydrothieno[2,3-c]isoquinolin -2-yl)ureido)-N- (thiazol-2-yl)benzenesulfanamide (10g)}

Obtained from carboazide 8 and sulfathiazole. The solid product was recrystallized from dioxane as white $\mathrm{crystals}$ IR $\mathrm{v}\left(\mathrm{cm}^{-1}\right)$ : 3400, 3350, 3250 (3NH), 3030 (CH aromatic), 2920, 2850 (CH aliphatic), 1705 (CONH). ${ }^{1} \mathrm{H}-\mathrm{NMR}$ (DMSO-d $): 1.70$ (m, $4 \mathrm{H}, 2 \mathrm{x}$ $\mathrm{CH}_{2}$ cyclohexeno), $2.60\left(\mathrm{~m}, 4 \mathrm{H}, 2 \times \mathrm{CH}_{2}\right.$ cyclohexeno), $3.20\left(\mathrm{~m}, 4 \mathrm{H}, 2 \times \mathrm{CH}_{2}-\mathrm{N}\right.$ morpholine $), 3.85\left(\mathrm{~m}, 4 \mathrm{H}, 2 \times \mathrm{CH}_{2}-\mathrm{O}\right.$ morpholine), 6.30 (s, $2 \mathrm{H}, 2 \times \mathrm{CH}=\mathrm{C}$ pyrrolyl), $6.70(\mathrm{~s}, 2 \mathrm{H}, 2 \mathrm{CH}-\mathrm{N}$ pyrrolyl), 7.20-7.80 (m, $6 \mathrm{H}, \mathrm{ArH}+2 \mathrm{CH}$ thiazole), 8.90 (NHph), 9.60 (NHCO), 10.60 (s, 1H, NH thiazole).

\section{8-Morpholin-4-yl-9,10,11,12-tetrahydropyrrolo[1'",2":4',5']pyrazino[2',3' :5,4] thieno[2,3-c] isoquinolin-4(5H)-one (11)}

A suspension of caboazide compound $8(1 \mathrm{~g}, 2.45 \mathrm{mmol})$ in xylene $(5 \mathrm{ml})$ was refluxed for $2 \mathrm{hrs}$. The solid product which formed during reflux was filtered off, dried and recrystallized from dioxane into white crystals in $38 \%$ yield, m.p. $320-$ $322^{\circ} \mathrm{C}$. Anal. Calcd. For: $\mathrm{C}_{20} \mathrm{H}_{20} \mathrm{~N}_{4} \mathrm{O}_{2} \mathrm{~S}(380.47) \mathrm{C}, 63.14 ; \mathrm{H}, 5.30 ; \mathrm{N}, 14.73 ; \mathrm{S}, 8.43 \%$. Found: C, 63.22; H, 5.38; N, 14.86; $\mathrm{S}, 8.50 \%$. IR v $\left(\mathrm{cm}^{-1}\right): 3280(\mathrm{NH}), 2920,2850$ ( $\mathrm{CH}$ aliphatic), 1640 (CO pyrazine), $1585(\mathrm{C}=\mathrm{N}) .{ }^{1} \mathrm{H}-\mathrm{NMR}\left(\mathrm{CF}_{3} \mathrm{CO}_{2} \mathrm{D}\right): 1.80$ (m, 4H, $2 \times \mathrm{CH}_{2}$ cyclohexeno), 2.50 (m, 4H, $2 \times \mathrm{CH}_{2}$ cyclohexeno), 3.65 ( $\mathrm{m}, 4 \mathrm{H}, 2 \mathrm{CH} 2-\mathrm{N}$ morpholine), 4.20 ( $\mathrm{m}, 4 \mathrm{H}, 2 \times$ $\mathrm{CH}_{2}-\mathrm{O}$ morpholine), 6.20 (s, 1H, CH C:2 pyrrole), 6.50 (s, 1H, CH C:1 pyrrole), 7.30 (s, $1 \mathrm{H}, \mathrm{CH} \mathrm{C}: 3$ pyrrole).

\section{4-Chloro-8-morpholin-4-yl-9,10,11,12-tetrahydropyrrolo[1",2":4',5'] pyrazino[2',3':5,4]thieno[2,3-c]isoquinoline (12)}

A solution of pyrrolopyrazinothienoisoquinolinone $11(1.00 \mathrm{~g}, 2.50 \mathrm{mmol})$ in phosphorus oxychloride $(3 \mathrm{ml})$ was refluxed for $2 \mathrm{hrs}$. The solid precipitate which formed on cooling and dilution with water was filtered off, dried and recrystallized from ethanol into green crystals in $60 \%$, m.p. $116-118^{\circ} \mathrm{C}$. Anal. Calcd. For: $\mathrm{C}_{20} \mathrm{H}_{19} \mathrm{CIN}_{4} \mathrm{OS}(398.92) \mathrm{C}, 60.22 ; \mathrm{H}, 4.80 ; \mathrm{N}, 14.04$; S, 8.04\%. Found: C, 60.30; H, 5.00; N, 13.92; S, 8.15\%. IR v $\left(\mathrm{cm}^{-1}\right): 2920,2850$ (CH aliphatic), 1620 (C=N). ${ }^{1} \mathrm{H}-\mathrm{NMR}$ $\left(\mathrm{CDCl}_{3}\right): 1.70\left(\mathrm{~m}, 4 \mathrm{H}, 2 \times \mathrm{CH}_{2}\right.$ cyclohexeno), $2.60\left(\mathrm{~m}, 4 \mathrm{H}, 2 \times \mathrm{CH}_{2}\right.$ cyclohexeno), $3.25\left(\mathrm{~m}, 4 \mathrm{H}, 2 \times \mathrm{CH}_{2}-\mathrm{N}\right.$ morpholine), 3.80 (m, 4H, $2 \times \mathrm{CH}_{2}-\mathrm{O}$ morpholine), 6.35 (s, 1H, CH C:2 pyrrole), 6.90 (s, $1 \mathrm{H}, \mathrm{CH} \mathrm{C:3} \mathrm{pyrrole),} 7.20$ (s, $1 \mathrm{H}, \mathrm{CH} \mathrm{C:1}$ pyrrole).

\section{4-Phenylamino-8-morpholin-4-yl-9,10,11,12-tetrahydropyrrolo[1",2" $\left.: 4^{\prime}, 5^{\prime}\right]$ pyrazino[2',3':5,4]thieno[2,3-c]isoquinoline (13)}

A mixture of chloro compound $12(0.5 \mathrm{~g}, 1.26 \mathrm{mmol})$ and aniline $(0.25 \mathrm{ml}, 2.70 \mathrm{mmol})$ was heated under neat conditions for 5 minutes then ethanol $(15 \mathrm{ml})$ was added and reflux was continued for additional $2 \mathrm{hrs}$. The solid product which formed on cooling was filtered off, dried and recrystallized from ethanol: dioxane mixture into white crystals in $74 \%$ yield, m.p. $262-264^{\circ} \mathrm{C}$. Anal. Calcd. For: $\mathrm{C}_{26} \mathrm{H}_{25} \mathrm{~N}_{5} \mathrm{OS}(455.59) \mathrm{C}, 68.55 ; \mathrm{H}, 5.53 ; \mathrm{N}, 15.37 ; \mathrm{S}, 7.04 \%$. Found: $\mathrm{C}, 68.60 ; \mathrm{H}, 5.48$; $\mathrm{N}, 15.44 ; \mathrm{S}, 7.20 \%$. IR v $\left(\mathrm{cm}^{-1}\right): 3400(\mathrm{NH}), 3030\left(\mathrm{CH}\right.$ aromatic) 2920, 2850 (CH aliphatic), $1595(\mathrm{C}=\mathrm{N}) .{ }^{1} \mathrm{H}-\mathrm{NMR}$ (DMSO$\mathrm{d}_{6}$ ): $1.70\left(\mathrm{~m}, 4 \mathrm{H}, 2 \times \mathrm{CH}_{2}\right.$ cyclohexeno), 2.70 (m, 4H, $2 \times \mathrm{CH}_{2}$ cyclohexeno), 3.20 (m, 4H, $2 \times \mathrm{CH}_{2}-\mathrm{N}$ morpholine), 3.80 (m, 
4H, 2x $\mathrm{CH}_{2}-\mathrm{O}$ morpholine), 6.35 (s, $1 \mathrm{H}, \mathrm{CH} \mathrm{C:2} \mathrm{pyrrole),} 6.90$ (s, $1 \mathrm{H}, \mathrm{CH} \mathrm{C:3} \mathrm{pyrrole),} \mathrm{7.20-7.80} \mathrm{(m,} 6 \mathrm{H}, \mathrm{ArH}+\mathrm{CH} \mathrm{C}: 1$ pyrrole), $9.70(\mathrm{~s}, 1 \mathrm{H}, \mathrm{NH})$.

\section{4-Hydrazino-8-morpholin-4-yl-9,10,11,12-tetrahydropyrrolo[1",2":4',5'] pyrazino[2',3':5,4]thieno[2,3-c]isoquinoline (14)}

A solution of chloro compound (13) $(0.50 \mathrm{~g}, 1 \mathrm{mmol})$ and hydrazine hydrate $(0.25 \mathrm{~g}, 5 \mathrm{mmol})$ in ethanol was refluxed for 2 hrs. The solid product formed on cooling was filtered off, dried and recrystallized from ethanol into white crystals in $52 \%$ yield, m.p. $242-244^{\circ} \mathrm{C}$. Anal. Calcd. For: $\mathrm{C}_{20} \mathrm{H}_{22} \mathrm{~N}_{6} \mathrm{OS}(394.50) \mathrm{C}, 60.89 ; \mathrm{H}, 5.62 ; \mathrm{N}, 21.30$; S, 8.13\%. Found: $\mathrm{C}, 60.97 ; \mathrm{H}$, 5.58; N, 21.38; S, 8.25\%. IR v (cm-1): 3350, 3300, $3250\left(\mathrm{NH}, \mathrm{NH}_{2}\right), 2920,2850$ (CH aliphatic), $1640(\mathrm{C}=\mathrm{N}) .{ }^{1} \mathrm{H}-\mathrm{NMR}$ $\left(\mathrm{CDCl}_{3}\right): 1.80\left(\mathrm{~m}, 4 \mathrm{H}, 2 \times \mathrm{CH}_{2}\right.$ cyclohexeno), $2.70\left(\mathrm{~m}, 4 \mathrm{H}, 2 \times \mathrm{CH}_{2}\right.$ cyclohexeno), 3.25 (m, 4H, $2 \times \mathrm{CH}_{2}-\mathrm{N}$ morpholine), $\left.3.85\left(\mathrm{~m}, 4 \mathrm{H}, 2 \times \mathrm{CH}_{2}-\mathrm{O} \text { morpholine), } 6.35 \text { (s, } 1 \mathrm{H}, \mathrm{CH} \mathrm{C:2} \mathrm{pyrrole),} 6.50 \text { (s, 2H, NH}\right)_{2}\right), 6.85$ (s, $1 \mathrm{H}, \mathrm{CH} \mathrm{C}: 3$ pyrrole), 7.20 (s, $1 \mathrm{H}, \mathrm{CH} \mathrm{C:1}$ pyrrole), $7.90(\mathrm{~s}, 1 \mathrm{H}, \mathrm{NH})$.

\section{Benzylidene-8-morpholin-4-yl-9,10,11,12-tetrahydropyrrolo[1",2":4',5'] pyrazino[2',3':5,4]thieno[2,3-c]isoquinoline-4-ylhydrazide (15)}

A mixture of hydrazine compound $14(0.50 \mathrm{~g}, 1.27 \mathrm{mmol})$ and benzaldehyde $(0.5 \mathrm{ml}, 4.7 \mathrm{mmol})$ was heated under neat conditions for 5 minutes then ethanol $(10 \mathrm{ml})$ was added and reflux was continued for 2 hrs. The solid product formed on cooling was filtered off, dried and recrystallized from ethanol: dioxane mixture as yellow crystals in $75 \%$ yield. Anal. Calcd. For: $\mathrm{C}_{27} \mathrm{H}_{26} \mathrm{~N}_{6} \mathrm{OS}(482.61) \mathrm{C}, 67.20 ; \mathrm{H}, 5.43 ; \mathrm{N}, 17.41 ; \mathrm{S}, 6.64 \%$. Found: C, 67.12; H, 5.50; N, 17.26; S, 6.85. IR v (cm $\left.{ }^{-1}\right)$ : $3030\left(\mathrm{CH}\right.$ aromatic), $1640(\mathrm{C}=\mathrm{N}) .{ }^{1} \mathrm{H}-\mathrm{NMR}\left(\mathrm{CF}_{3} \mathrm{CO}_{2} \mathrm{D}\right): 1.80\left(\mathrm{~m}, 4 \mathrm{H}, 2 \times \mathrm{CH}_{2}\right.$ cyclohexeno), $2.75\left(\mathrm{~m}, 4 \mathrm{H}, 2 \times \mathrm{CH}_{2}\right.$ cyclohexeno), 3.20 (m, 4H, $2 \times \mathrm{CH}_{2}-\mathrm{N}$ morpholine), 3.85 (m, 4H, $2 \times \mathrm{CH}_{2}-\mathrm{O}$ morpholine), 6.35 (s, $\left.1 \mathrm{H}, \mathrm{CH} \mathrm{C}: 2 \mathrm{pyrrole}\right), 6.95$ (s, $1 \mathrm{H}, \mathrm{CH}$ C:3 pyrrole), 7.20-7.80 (m, 7H, ArH + CHph + $\mathrm{CH} \mathrm{C:1} \mathrm{pyrrole).}$

\section{0-Morpholin-4-yl-11,12,13,14-tetrahydro[1,2,4]triazolo[3"',4"':6',1'] pyrrolo[1",2":4',5']pyrazino[2',3':5,4]thieno[2,3-c]isoquinoline (16)}

A mixture of hydrazino compound $15(0.5 \mathrm{~g}, 1 \mathrm{mmol})$ and triethylortho formate $(2 \mathrm{ml})$ in presence of glacial acetic acid $(0.5$ $\mathrm{ml}$ ) was refluxed for $2 \mathrm{hrs}$. The solid product which formed during reflux was filtered off, dried and recrystallized from ethanol: dioxane mixture as white crystals in $74 \%$ yield, m.p. $332-334^{\circ} \mathrm{C}$. Anal. Calcd. For: $\mathrm{C}_{21} \mathrm{H}_{20} \mathrm{~N}_{6} \mathrm{OS}(404.50) \mathrm{C}$, 62.36; H, 4.98; N, 20.78; S, 7.93\%. Found: C, 62.45; H, 5.10; N, 20.63; S, 8.10\%. IR v (cm $\left.{ }^{-1}\right)$ : 2950, 2850 (CH aliphatic), 1640 $(\mathrm{C}=\mathrm{N}) .{ }^{1} \mathrm{H}-\mathrm{NMR}\left(\mathrm{CF}_{3} \mathrm{CO}_{2} \mathrm{D}\right): 1.80\left(\mathrm{~m}, 4 \mathrm{H}, 2 \times \mathrm{CH}_{2}\right.$ cyclohexeno), $2.75\left(\mathrm{~m}, 4 \mathrm{H}, 2 \times \mathrm{CH}_{2}\right.$ cyclohexeno), $3.30(\mathrm{~m}, 4 \mathrm{H}, 2 \times$ $\mathrm{CH}_{2}-\mathrm{N}$ morpholine), 3.95 (m, 4H, $2 \times \mathrm{CH}_{2}-\mathrm{O}$ morpholine), 6.35 (s, $1 \mathrm{H}, \mathrm{CH} \mathrm{C:2} \mathrm{pyrrole),} 6.90$ (s, 1H, $\mathrm{CH} \mathrm{C:3}$ pyrrole), 7.20 (s, $1 \mathrm{H}, \mathrm{CH} \mathrm{C:1}$ pyrrole), $8.30(\mathrm{~s}, 1 \mathrm{H}, \mathrm{CH}$ triazole).

\section{REFERENCES:}

[1] Bentley, K. W. Nat. Prod. Rep. 2005, 22, 249-268. $\beta$-Phenylethylamines and the isoquinoline alkaloids

[2] Scott, J. D., and Williams, R. M. Chem. Rev. 2002, 102, 1669-1730. Chemistry and Biology of the Tetrahydroisoquinoline Antitumor Antibiotics.

[3] Iwasa, K., Moriyasu, M., Yamori, T., Turuo, T., Lee, D.-U, and Wiegrebe, W.J. Nat. Prod. 2001, 64, 896-898. In Vitro Cytotoxicity of the Protoberberine-Type Alkaloids

[4] Iwasa, K., Moriyasu, M., Tachibana, Y., Kim, H.S., Wataya, Y., Wiegrebe, W., Bastow, K. F., Cosentino, L. M., Kozuka, M., and Lee, K.H. Bioorg. Med. Chem. 2001, 9, 2871-2884. Simple Isoquinoline and Benzylisoquinoline Alkaloids as Potential Antimicrobial, Antimalarial, Cytotoxic, and Anti-HIV Agents

[5] Cheng, P., Huang, N., Jiang, Z. Y., Zhang, Q., Zheng, Y. T., Chen, J. J., Zhang, X. M., and Ma, Y.B. Bioorg. Med. Chem. Lett. 2008, 18, 2475-2478. 1- Aryltetrahydroisoquinoline analogs as active anti-HIV agents in vitro.

[6] Pham, V.C., Ma, J., Thomas, S. J., Xu, Z., and Hecht, S.M. J. Nat. Prod. 2005, 68, 1147- 1152. Alkaloids from Alangium javanicum and Alangium grisolleoides that Mediate $\mathrm{Cu}^{2+}$ - Dependent DNA Strand Scission.

[7] Naito, R., Yonetoku, Y., Okamoto, Y., Toyoshima, A., Ikeda, K., and Takeuchi, M. J Med. Chem. 2005, 48, 65976606.Synthesis and Antimuscarinic properties of Quinclidin-3-yl- 1,2,3,4-Tetrahydroisoquinoline-2-carboxylate Derivatives as Novel Muscarinic Receptor Antagonists

[8] Leeson, P. D., Carling, R. W., Moore, K. W., Moseley, A. M., Smith, J. D., Stevenson, G., Chan, T., Baker, R., Foster, A. C., Grimwood, S., Kemp, J. A., Marshall, G. R., and Hoogsteen, K., J. Med. Chem. 1992, 35, 1954-1968. 4Amido-2 carboxytetrahydroquinolines. Structure-activity relationships for antagonism at the glycine site of the NMDA receptor.

[9] Nagata, R., Tanno, N., Kodo, T., Ae, N., Yamaguchi, H., Nishimura, T., Antoku, F., Tatsuno, T., Kato, T., Tanaka, Y. and Nakamura, M., J. Med. Chem. 1994, 37, 3956-3968. Tricyclic Quinoxalinediones:5,6-Dihydro-1H-pyrrolo[1,2,3de]quinoxaline- 2,3-diones and 6,7-Dihydro-1H,5H-pyrido[1,2,3-de]quinoxaline-2,3-diones as Potent Antagonists for the Glycine Binding Site of the NMDA Receptor. 
[10] Guo, F., Chang, B. H. and Rizzo, C. J., Bioorg. Med. Chem. Lett. 2002, 12, 151-154. An N1-Hydrogen bonding model for flavin coenzyme.

[11] Stanton, J. L. and Ackerman, M. H. J. Med. Chem. 1983, 26, 986-989. Synthesis and anticonvulsant activity of some tetracyclic indole derivatives.

[12] Shutske, G. M., and Kapples, J. U.S. Pat., 1998, 4, 753, 950; Chem. Abstr. 1988, 109, 128990j.

[13] McCarthy, J. R. and Winder, P. J. Eur. Pat. 1985, 126, 487, Chem. Abstr., 1985, 102, 135078r.

[14] Hashimoto K., Inoe, M., Tomoyasu, T., Kamisako, T., Sugimoto, Y. and Kuwabara, T., Jap. Pat., 1994, 06, 92, 963; Chem Abstr., 1994, 121, 157630v.

[15] Sauter, F., Jordis, U., Tanyolac, S. and Martinek, P. Arch. Pharm. 1988, 321, 241-246. Synthesen neuer ChinolonChemotherapeutika, 2. Mitt.: Thieno[3,2-g]chinolin-und [1]Benzothieno-[5,6,7-ij]chinolizincarbonsäurederivate.

[16] Wagner, G., Vieweg, H. and Leistner, S. Phramazie 1993, 48, 576-578.

[17] Kamal El-Dean, A. M., Radwan, Sh. M. and Zaki, R.M., J. Chin. Chem. Soc. 2008, 55, 1-10. Synthesis of Morphlinotetrahydrothieno[2,3-c]isoquinolines.

[18] Kamal, A.M., Radwan, Sh.M., and Zaki, R.M. J. Chem. Res. 2010, 34, 596-602 Reactions of 1-amino-5-morpholino6,7,8,9-tetrahydrothieno [2,3-c]isoquinoline-2-carbonitrile.

[19] Kamal, A.M, Radwan, Sh.M., and Zaki, R. M. Eur. J. Med. Chem. 2011, 46, 567-578. Synthesis and biological activity of pyrazolothienotetrahydroisoquinoline and [1,2,4]triazolo[3,4-a]thienotetrahydroisoquinoline derivatives.

[20] Kamal, A.M., Radwan, Sh.M. and Zaki, R. M. J. Chin. Chem. Soc. 2011, 58, 544-554. Synthesis and Reactions of 1 Amino-5-morpholin-4-yl-6,7,8,9-tetrahydro thieno[2,3-c]isoquinoline.

[21] Zaki, R.M., Kamal El-Dean, A.M.and Radwan, Sh.M. Afindad 2012, LXVII, 424-434. Synthesis and reactions of novel thienotetrahydroisoquinoline compounds. 